Elsevier Editorial System(tm) for Colloids and Surfaces A: Physicochemical and Engineering Aspects

Manuscript Draft

Manuscript Number: COLSUA-D-16-02286R1

Title: Dynamics of a flexible fibre in a sheared two-dimensional foam: numerical simulations

Article Type: SI: Eufoam 2016

Keywords: fibre-laden foam

papermaking

tumbling motion

bubble model

Corresponding Author: Dr. Vincent J. Langlois, Dr

Corresponding Author's Institution: Université Claude Bernard Lyon 1

First Author: Vincent J. Langlois, Dr

Order of Authors: Vincent J. Langlois, Dr; Stefan Hutzler, Pr.

Manuscript Region of Origin: FRANCE

Abstract: Recently there has been a renewed interest in using foamy suspensions of wood fibres as a carrier fluid in papermaking but there is a lack of fundamental understanding of the dynamics of such a three-phase system.

In this article we propose a numerical model for the dynamics of an individual flexible fibre within a flowing foam, based

on discrete-element methods. As is observed in a Newtonian shear flow, we observe that the fibre systematically experiences a tumbling instability: the disordered motion of bubbles

cannot prevent the pseudo-periodical flip of the fibre. Our simulations show that the tumbling time decreases almost as the inverse of the strain rate. It also decays when the fibre

length is increased, though for long enough fibres it reaches a constant value. Similarly the tumbling time is also surprisingly independent of the stiffness of the fibre.

Because of their tumbling motion, long and flexible fibres spend most of the time in a coiled geometry. This would imply that using foam as a carrier fluid is not enough

to keep fibres aligned with the flow. However, further refinements of the model will need to be considered to arrive at firm conclusions regarding alignment. 


\section{Dear Editor,}

We would like to thank the referee for his careful reading and constructive remarks. We tried to follow his recommendations, and to answer his comments and concerns regarding the article. The modifications we brought to the paper are listed below:

\section{Reviewer \#1}

* General questions that seem not to be addressed are: Is there shear-banding or localisation? Does it matter where between the side walls the fibre is placed? (I guess it shouldn't, but I presume that this was checked.)

Since no additional wall friction is added (see our previous study of the bubble model in Langlois et al. [2008]), no shear-banding is observed. The average velocity profile is mostly undisturbed by the presence of a unique fibre, and remains linear.

The initial $y$-position of the fibre is of no influence, except in the 'pathological' case where it comes in contact with one of the walls and remains there.

* lines 10-20 are not very clear on how the presence of the fibres affects these foam properties; it would be useful to expand this section to give the reader more of a feel for the complexity of this area of research and the way in which a foam interacts with fibres.

We have added to the introduction a more thorough description on the effects of fibres on the foam, recently observed experimentally (1.15 to 21 ).

* lines 63 and 96: I find the use of the word "spherical" misleading, since in a 2D experiment bubbles are more discoidal than spherical and they are certainly treated here as discs. Once the description of the bubble model is restricted to 2D, I suggest to use "discs" exclusively.

In the simulations the bubbles are indeed treated as disks, though we had initially used the term "spheres" since these disks are meant to mimick a bubble raft made of roughly spherical bubbles seen from above. However we agree that this can lead to some confusion and corrected this to use exclusively 'disks'.

* line 68/71: W needs to be defined earlier, i.e. at line 68 not 71.

We modified the description of the geometrical properties to make them clearer (1.72 to 76 ).

* fig 1 (and the graphical abstract) shows a situation in which two non-adjacent fibre particles are touching. This is not mentioned in the text, but raises interesting questions: is this behaviour typical or (as fig 5 suggests) unusual (making it a poor choice for the graphical abstract)? Does such a situation change the speed with which the fibre tumbles? What is the interaction, if any, between non-adjacent fibre particles?

This type of collision can happen, but mostly for not very rigid fibres. Such a collision between two non-adjacent fibre particles is treated as a bubble-bubble collision (that is, no attractive force), as explained lines 117-119.

* lines 85-86: what is "the" foam. If I understand correctly, the authors need to make clear that here they mean "a" real foam, not the one in the simulations.

This is indeed what we meant; we added this precision (1.91).

* lines 100-105: It would help to have an explanation of what equations (4) and (5) mean, rather than expecting the reader to infer how they work, i.e. please expand upon their description as an "elastic force" and a "bending force".

The elastic force is effectively a simple spring between adjacent particles, which tends to restore their distance to its equilibrium value $l_{0}$. The bending force tends to realign each triplet of adjacent particles. We added these precisions to the correspondong paragraph (1.114-121). 
* lines 107-108: is it the case that this interaction has both an elastic and a viscous component? This should be explicit. And why are the same $\kappa$ and $c_{b}$ used here?

We added this precision. The same values were chosen for sake of simplicity and to avoid having too many control parameters. However, in the regime we are most interested in, the fibre remains rather rigid and very few of these collisions happen.

* equations (6) and (7): is it assumed that the elastic force between adjacent particles is zero in order to get the rest length $(N-1) l_{0}$ ? Surely this is rarely exact?

The factor $(N-1) l_{0}$ is indeed the equilibrium length of the fibre, but is only used here to renormalize the actual spatial extension of a fibre at any given time, when it is not at equilibrium.

* fig 4: I found it hard to distinguish the two lines.

We have attempted to improve the difference between both curves.

* fig 6: can the authors make a conjecture about what sets $\tau_{0}=20$ ? Can they propose an explanation of why $\tau_{r}$ decreases with $N$ ? As mentioned above, this paper is currently mostly a list of results, but I think it could be made stronger with more explanations of this kind. * $\$ 3.4$ : surely this section requires a comment on the result in eq. (10): the exponent on $S$ is very small, and within the error bars I could draw a horizontal line, so the influence of stiffness seems weak. In particular, the statement in the conclusions that stiffness has an effect on the tumbling motion seems bold. ${ }^{*} \S 3.5$ : the results give the impression that the tumbling always occurs, but that for a wide gap there are long periods without tumbling. These seem hard to reconcile, hence the authors' use of "abnormally". Is this the only choice of parameters for which tumbling doesn't happen?

Section 3.4 has been greatly expanded and now includes an additional figure. We performed additional simulations and conclude that indeed the effect of stiffness on the tumbling time can be considered as negligible. We have now included a more detailed discussion for the constant value of the tumbling time for long enough fibres, which we predict from the dynamical shape of the fibre and compare to theoretical predictions.

* the references need tidying up, for example to insert missing journal volumes.

The lacking information has been added to the bibliography.

\section{References}

V. J. Langlois, S. Hutzler, and D. Weaire. Rheological properties of the soft-disk model of twodimensional foams. Phys. Rev. E, 78:021401, 2008. doi: 10.1103/PhysRevE.78.021401. 

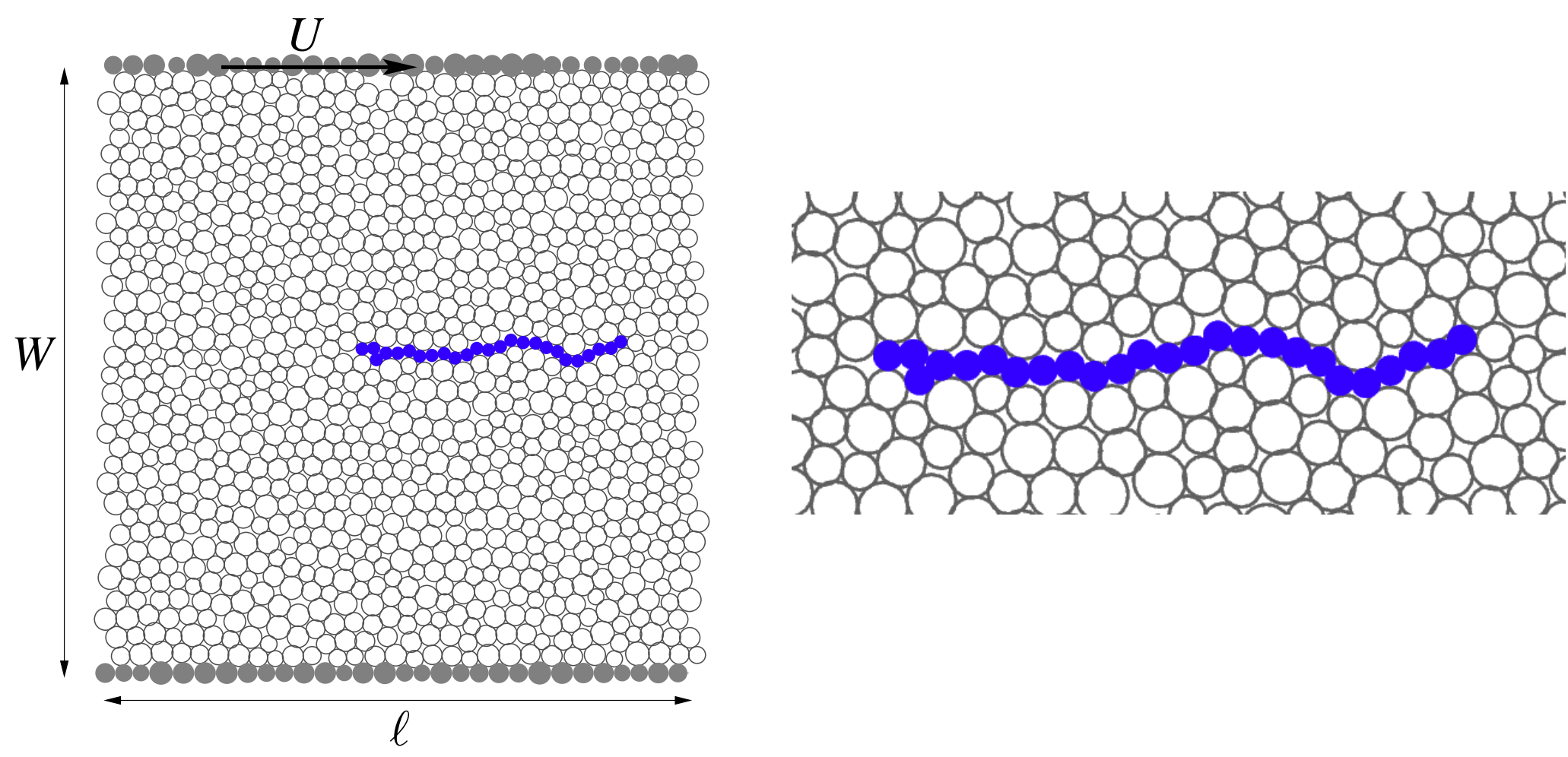
${ }^{*}$ Highlights (for review) 


\title{
Dynamics of a flexible fibre in a sheared two-dimensional foam: numerical simulations
}

\author{
Vincent J. Langlois ${ }^{\mathrm{a}, *}$, Stefan Hutzler ${ }^{\mathrm{b}}$ \\ ${ }^{a}$ Laboratoire de Géologie de Lyon, Université Claude Bernard Lyon 1 - ENS de Lyon- \\ CNRS, 2 rue R. Dubois, 69100 Villeurbanne, France \\ ${ }^{b}$ School of Physics, Trinity College Dublin, The University of Dublin, Ireland.
}

\begin{abstract}
Recently there has been a renewed interest in using foamy suspensions of wood fibres as a carrier fluid in papermaking but there is a lack of fundamental understanding of the dynamics of such a three-phase system. In this article we propose a numerical model for the dynamics of an individual flexible fibre within a flowing foam, based on discrete-element methods. As is observed in a Newtonian shear flow, we observe that the fibre systematically experiences a tumbling instability: the disordered motion of bubbles cannot prevent the pseudo-periodical flip of the fibre. Our simulations show that the tumbling time decreases almost as the inverse of the strain rate. It also decays when the fibre length is increased, though for long enough fibres it reaches a constant value. Similarly the tumbling time is also surprisingly independent of the stiffness of the fibre. Because of their tumbling motion, long and flexible fibres spend most of the time in a coiled geometry. This would imply that using foam as a carrier fluid is not enough to keep fibres aligned with the flow. However, further refinements of the model will need to be considered to arrive at firm conclusions regarding alignment.
\end{abstract}

Keywords: Fibre-laden foams, papermaking, tumbling instability, bubble model.

\section{Introduction}

The possibility of replacing water by a liquid foam as the carrier fluid for wood fibres in papermaking has led to a renewed interest in the technique by the paper industry. Although this idea was initially proposed in the 1970s (Smith 5 et al., 1974), quantitative investigations of the behaviour of fibre-laden foams have only begun quite recently. The use of a fibre-laden foam would considerably reduce the water consumption and consequently the energy needed for the drying

\footnotetext{
${ }^{*}$ Corresponding author

Email address: vincent.langlois@univ-lyon1.fr (Vincent J. Langlois)
} 
of the paper. Furthermore, the technique might enable improvement and better control of the properties of the final fibre network: the same method could then be applied to the manufacturing of other novel fibrous materials (e.g. for insulation, non-woven textiles, oil absorption, etc.).

In the papermaking framework, several experimental studies (Al-Qararah et al., 2013, 2015b; Jäsberg et al., 2015; Haffner et al., 2017) have recently investigated the influence of the fibres (depending on their physico-chemical 15 characteristics) on the properties of the wet foam used as carrier fluid: incorporating fibres while mixing the foaming liquid produces a foam with smaller bubbles and a higher liquid fraction than without fibres. Foam viscosity has been found to increase with increased content of rough wood fibres, although the foam still remains shear-thinning. This effect might not be observed when 20 using artificial smooth fibres such as viscose. Further work also addressed the alteration of local foam geometry in the presence of fibres and the slow-down in foam coarsening (Whyte et al., 2017). The influence of the foam on the properties of the final dry fibre network has been studied by Al-Qararah et al. (2015a), who showed that the pore size distribution in the paper sheet is more regular

25 if the fibres are deposited from a foam suspension rather than from classical water-based pulp.

Therefore it is important to investigate theoretically and numerically the dynamics of fibre-laden foams, in order to better understand the key parameters that control the interactions between fibres and bubbles: how does the presence 30 of fibres affect the properties of the foam, and how can we use the foam carrier in order to tune the properties of the final fibre network?

In the past 20 years, the physics of liquid foams has known flourishing progress (Weaire and Hutzler, 1999; Cantat et al., 2013), whether it deals with 35 the physico-chemical properties of the thin liquid films, the quasi-static properties of a foam (bubble coarsening and ripening, drainage) or its dynamical behaviour (influence of flow on topology, rheology). Also, since the pioneering theoretical works of Jeffery (1922) on the dynamics of ellipsoidal particles, the study of fibres in Newtonian fluid flows has been the subject of many experimen-

40 tal and numerical studies, with motivations as diverse as papermaking, water purification, dynamics of DNA molecules or microswimmers (Forgacs and Mason, 1959; Yamamoto and Matsuoka, 1996; Ross and Klingenberg, 1997; Switzer and Klingenberg, 2003; Subramanian and Koch, 2005; Gauger and Stark, 2006; Lindström and Uesaka, 2007; Wandersman et al., 2010; Lindner and Shelley, 45 2015; Farutin et al., 2016). In particular, fibre suspensions have often been modelled by studying the interactions between a laminar simple shear or Poiseuille flow and flexible rods modelled as strings of spherical (or circular) beads.

Here we propose to combine such a model for fibres with a model that describes a foam itself as a packing of soft spheres or disks (in 2D). This so-called 50 bubble model, or soft-sphere model was introduced by Durian (1995) in order to simulate mechanical properties of wet foams. As shown by Langlois et al. (2008), this simplistic but computationally efficient approach is sufficient to reproduce the basic features of the rheology of foams: existence of a yield stress, 
Herschel-Bulkley shear-thinning rheology, occurrence of shear-bands in a Hele55 Shaw cell, and it can also be appropriate for more complex geometries (Langlois, 2014).

In this article we will consider the behaviour of a single fibre in a twodimensional (2D) foam under shear, as a preliminary study in order for further

${ }_{60}$ investigations of the rheology of three-dimensional fibre-laden foams. The article is organized as follows: in section 2 we describe the implementation of the bubble model and the modelling of the fibres; in section 3 we analyze the motion of the fibre as a function of its length and stiffness, strain rate and channel width. Finally we draw conclusions regarding the use of foam as a carrier fluid for fibres.

\section{Numerical model}

\subsection{Bubble model}

The 2D foam, as described by the bubble model (Durian, 1995; Langlois et al., 2008), is a dense packing of circular bubbles. A small polydispersity in

70 bubble size is introduced to prevent crystallization of the bubbles, with each bubble radius $R_{i}$ being chosen within a uniform distribution bounded by $R_{0} \times$ $(1 \pm 0.2), R_{0}$ being the average radius. The foam is produced by compressing a sample of 2,000 to 10,000 bubbles between two side-walls, of length $\ell=200 R_{0}$ and made of fixed bubbles. The final state is obtained for a gap $W$ between 75 these walls (see figure 1), defined by the packing fraction $\sum \pi R_{i}{ }^{2} /(W \ell)=0.90$ (the overlaps between bubbles being neglected). This corresponds to an effective liquid fraction $\phi=0.10$. One of the side-walls is then moved tangentially at a constant speed $U$, which defines the average strain rate as $\dot{\gamma}=U / W$. The other side-wall is kept stationary. The dynamics of the foam are computed by

so solving Newton's second law for each individual bubble, using classical numerical techniques originally developed for Molecular Dynamics (Pöschel and Schwager, 2005). Periodic boundary conditions are applied in the streamwise direction.

Bubbles interact with one another through elastic and viscous forces. When overlapping, two bubbles $i$ and $j$, located respectively at $\mathbf{r}_{i}$ and $\mathbf{r}_{j}$ and of radii

${ }_{85} R_{i}$ and $R_{j}$, repel each other via a linear elastic force. A bubble $j$ then exerts on bubble $i$ the force

$$
\mathbf{F}_{\mathbf{r}}^{i j}=-\kappa \frac{2 R_{0}}{R_{i}+R_{j}} \Delta_{i j} \mathbf{n}_{i j} .
$$

where $\kappa$ is the coefficient of elasticity (related to surface tension), $\mathbf{n}_{i j}$ is the unit normal vector between bubbles $i$ and $j$, defined by

$$
\mathbf{n}_{i j}=\frac{\mathbf{r}_{j}-\mathbf{r}_{i}}{\left|\mathbf{r}_{j}-\mathbf{r}_{i}\right|}
$$

and the overlap $\Delta_{i j}$ (see Fig.2) is given by $\Delta_{i j}=\left(R_{i}+R_{j}\right)-\left|\mathbf{r}_{j}-\mathbf{r}_{i}\right|$. The ratio 90 $2 R_{0} /\left(R_{i}+R_{j}\right)$ in equation (1) takes into account that larger bubbles are easier to deform than smaller ones. In a real flowing foam, energy is dissipated by the 

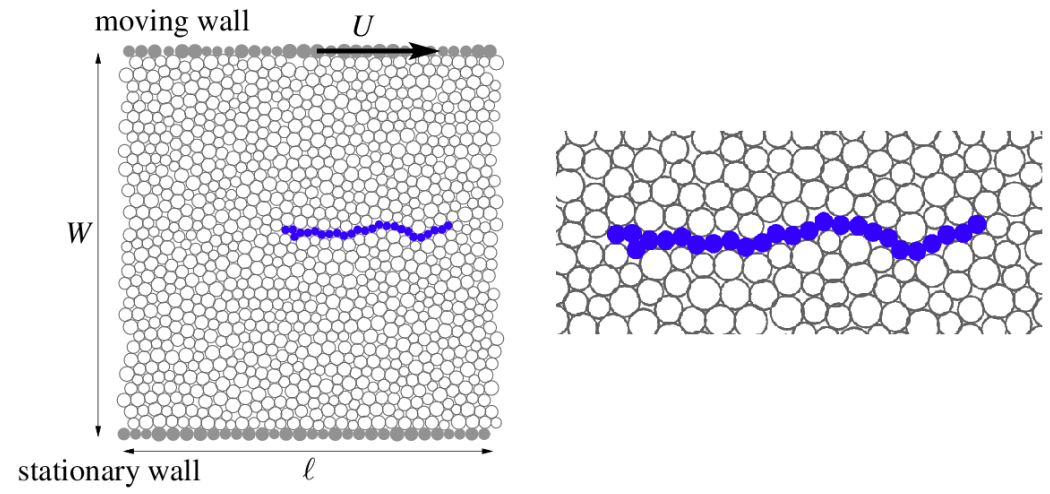

Figure 1: Foam produced by the bubble model, containing a fibre represented by 25 connected disks (appearing in blue), and close-up view of this fibre surrounded by bubbles. Fixed streamwise velocities ( 0 and $U$, respectively) are imposed on the bubbles composing both side-walls (full grey). Note that in the actual simulations the length of the cell is much longer ( $\ell$ about 100 bubble diameters).

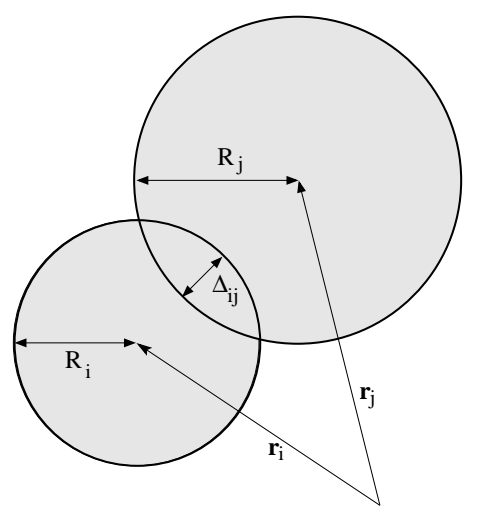

Figure 2: Overlap $\Delta_{i j}$ between two contacting bubbles of radii $R_{i}$ and $R_{j}$, located at $\mathbf{r}_{i}$ and $\mathbf{r}_{j}$, respectively. 
viscosity of the liquid within the films between bubbles. This is accounted for in the bubble model by introducing a viscous force $\mathbf{F}_{v}$ acting on a bubble $i$ in contact with a bubble $j$ :

$$
\mathbf{F}_{v}{ }^{i j}=c_{b}\left(\mathbf{v}_{j}-\mathbf{v}_{i}\right)
$$

where $c_{b}$ is a dissipation constant and $\mathbf{v}_{i}$ and $\mathbf{v}_{j}$ are the respective bubble velocities.

The two forces $\mathbf{F}_{\mathbf{r}}{ }^{i j}$ and $\mathbf{F}_{v}{ }^{i j}$ allow us to define the dimensionless Deborah number De $=\dot{\gamma} c_{b} / \kappa$, that relates the timescale of bubble dynamics $\tau_{b}=c_{b} / \kappa$ to the shear timescale $1 / \dot{\gamma}$.

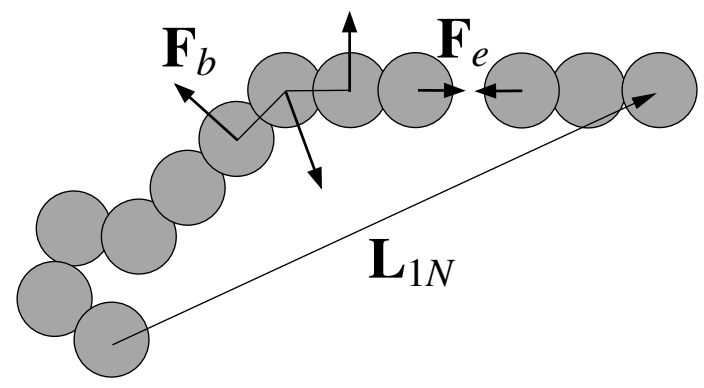

Figure 3: Elastic $\left(\mathbf{F}_{e}\right)$ and bending $\left(\mathbf{F}_{b}\right)$ forces experienced by the disks representing the fibre, whose shape is characterized by its end-to-end vector. Elastic forces tend to bring the fibre length back to equilibrium and bending forces tend to keep the fibre straightened.

Following the seminal works of Yamamoto and Matsuoka (1993), we model the deformable fibre as a string of $N$ disks of radius $R_{f}=0.6 R_{0}$. This model is particularly appropriate within the bubble model, since the dynamics of these fibre particles can be computed together with the dynamics of the bubbles. 105 Within the fibre, each particle exerts both an elastic and a bending force on its neighbours (see figure 3). Elasticity is modelled by adding linear springs between each pair of adjacent fibre particles. The elastic force experienced by a fibre particle $i$ is then

$$
\mathbf{F}_{e}^{i}=-\kappa\left(l_{i}-l_{0}\right) \mathbf{n}_{i-1, i}+\kappa\left(l_{i+1}-l_{0}\right) \mathbf{n}_{i, i+1}
$$

with $l_{i}=\left|\mathbf{r}_{i}-\mathbf{r}_{i-1}\right|$ and $l_{0}=1.8 R_{f}$ the distance between two fibre particles at 110 equilibrium. This force tends to bring the fibre back to its equilibrium length $L_{0}=(N-1) l_{0}$. Discretizing the bending free energy of a continuous elastic rod gives the following expression for the bending force acting on the particle $i$ 
(Gauger and Stark, 2006; Słowicka et al., 2012):

$$
\begin{aligned}
\mathbf{F}_{b}{ }^{i}= & S \times \kappa R_{0} \times\left\{\frac{\epsilon_{i-1}}{l_{i}} \mathbf{n}_{i-2, i-1}\right. \\
& -\left[\frac{\epsilon_{i-1}}{l_{i}} \mathbf{n}_{i-2, i-1} \cdot \mathbf{n}_{i-1, i}+\frac{\epsilon_{i}}{l_{i+1}}+\frac{\epsilon_{i}}{l_{i}} \mathbf{n}_{i-1, i} \cdot \mathbf{n}_{i, i+1}\right] \mathbf{n}_{i-1, i} \\
& +\left[\frac{\epsilon_{i}}{l_{i+1}} \mathbf{n}_{i-1, i} \cdot \mathbf{n}_{i, i+1}+\frac{\epsilon_{i}}{l_{i}}+\frac{\epsilon_{i+1}}{l_{i+1}} \mathbf{n}_{i, i+1} \cdot \mathbf{n}_{i+1, i+2}\right] \mathbf{n}_{i, i+1} \\
& \left.-\frac{\epsilon_{i+1}}{l_{i+1}} \mathbf{n}_{i+1, i+2}\right\}
\end{aligned}
$$

with $\epsilon_{i}=l_{0}$ if $2 \leq i \leq N-1$ and $\epsilon_{i}=0$ for $i=1$ or $N$. This force tends to restore alignment of each triplet of adjacent fibre particles, and therefore straightens the fibre. The parameter $S$ represents the dimensionless stiffness of the fibre. Finally, the interaction between a fibre particle and a bubble, or between two non-adjacent fibre particles, is treated as if it were a bubble-bubble collision (with repulsive and dissipative forces, see equations (1) and (3)). We

${ }_{120}$ will return to this treatment in section 4 where we discuss the modelling of fibre roughness.

In order to describe the shape of the fibre during the dynamics, we define its end-to-end vector $\mathbf{L}_{1 N}$ (as illustrated in figure 3), whose normalized components are noted as

$$
\Delta x(t)=\frac{x_{N}(t)-x_{1}(t)}{L_{0}} \quad \text { and } \quad \Delta y(t)=\frac{y_{N}(t)-y_{1}(t)}{L_{0}}
$$

where the indices 1 and $N$ correspond to both ends of the fibre. For instance, when the fibre is perfectly aligned in the streamwise $x$-direction, we have $\Delta y=0$, and if it is neither stretched nor compressed, $\Delta x=1$. Following Słowicka et al. (2015), we also define the fractional compression of the fibre as

$$
\alpha(t)=1-\frac{L(t)}{(N-1) l_{0}}
$$

with $L(t)=\left|\mathbf{L}_{1 N}\right|$ the absolute distance between the two ends of the fibre. $\alpha$ can be seen as a measurement of the state of the coiling of the fibre. When the fibre is coiled, we have $\alpha>0$, whereas at equilibrium $\alpha=0$ and when the fibre is stretched $\alpha<0$.

\subsection{Time integration}

${ }_{135}$ At a given iteration, all forces acting on each bubble/particle are computed. Overlaps between bubbles are found by using the linked cell algorithm (Pöschel and Schwager, 2005). As in previous implementations of the model (Langlois et al., 2008; Sexton et al., 2011; Langlois, 2014), an effective mass is assigned to each bubble/particle and we use the Verlet algorithm (of fourth order of 
precision) (Pöschel and Schwager, 2005) to compute the position of each bubble/particle at the next iteration from Newton's second law. The mass is chosen so that the motion of each bubble remains overdamped and inertia is therefore negligible in the dynamics: we set the ratio $\kappa m_{b} / c_{b}^{2}=1.5 \times 10^{-2}$. In order to compute accurately each collision between bubbles, the iterative timestep $\Delta t$ is chosen 100 times smaller than the characteristic viscous timescale:

$$
\Delta t=\frac{\tau_{v}}{100} \quad \text { with } \quad \tau_{v}=\frac{m_{b}}{c_{b}}
$$

\section{Dynamics of a fibre in a shear flow}

\subsection{Tumbling instability}

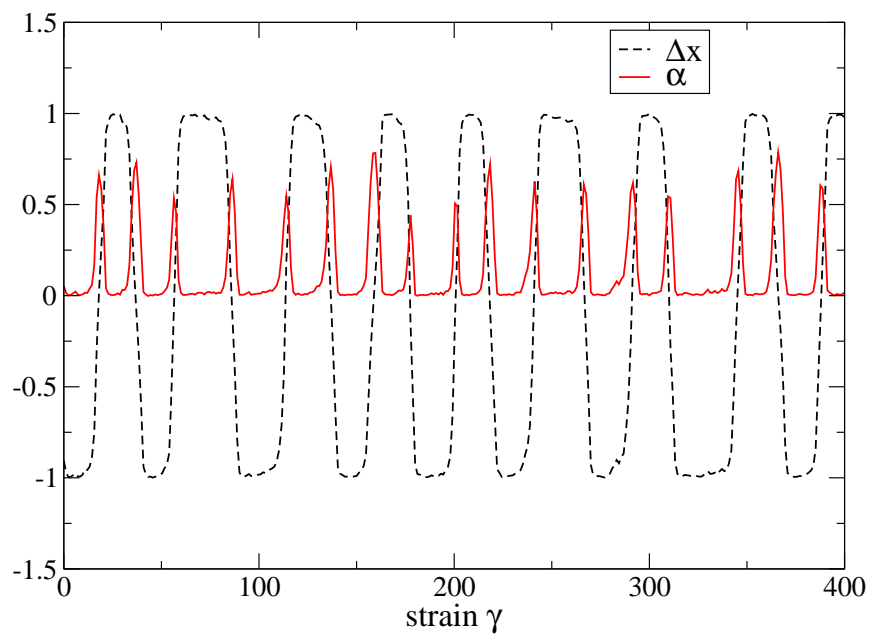

Figure 4: Normalized streamwise component $\Delta x / l_{0}$ of the end-to-end vector and fractional compression of the fibre as a function of strain, for $N=50, S=1.0$ and De $=1.0 \times 10^{-3}$. Most of the time the fibre is undeformed and oriented along the streamwise direction. Each peak in alpha corresponds to the fibre flipping (which results in reversing its direction).

Let us first remark that the presence of a single fibre does not affect the average linear velocity profile in the gap. We plot in figure 4 the streamwise extension $\Delta x$ of the fibre as a function of strain $\gamma=\dot{\gamma} \times t$, for a length $N=50$, a strain rate $\mathrm{De}=1.0 \times 10^{-3}$ and a stiffness $S=1.0$. In this example, we can observe that the fibre spends most of the time in a straight configuration $(|\Delta x| \simeq 1$ and $\alpha \simeq 0)$, during which it is roughly aligned with the direction of the flow. However, it also experiences successive flips, during which it rapidly coils $(\alpha>0.5)$ before straightening again in the opposite direction. This tumbling motion, which is also observed for an individual fibre within a viscous Newtonian flow (Słowicka et al., 2012, 2015), appears to be roughly periodic. Hence, the 
presence of the bubbles is not sufficient to channel the motion of fibre and prevent this instability. The snapshots in figure 5 illustrates the successive shapes taken by the fibre during one of these flips.

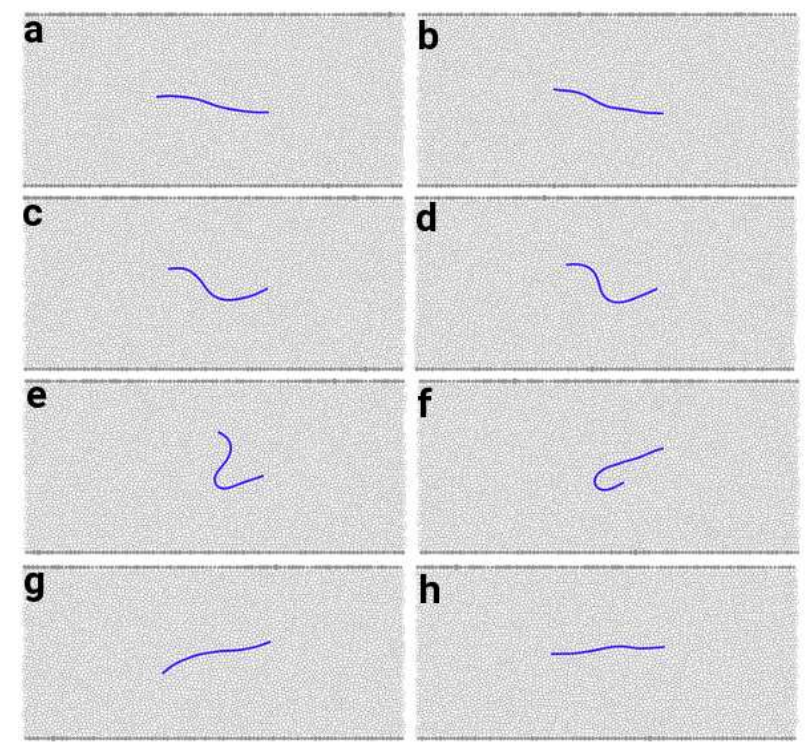

Figure 5: Successive snapshots of a fibre of length $N=50$ undergoing a 'flip'. The $x$-position of the middle particle of the fibre is kept at 0 in this representation. Images are separated by $\Delta \gamma=2$.

\subsection{Dependence on strain rate}

A fibre flip is identified by the rapid change in sign of $\Delta x$ and we mark its occurrence in time when $\Delta x=0$. The duration between two consecutive flips defines the tumbling time $T_{t}$. The average tumbling time for a given strain rate is computed over at least 50 flip events. Different from the case of a viscous shear flow in a Newtonian fluid, it is possible in a foam to define an internal timescale, independently of the strain rate. We therefore rescale the tumbling time with the internal timescale $\tau_{b}$, while varying the strain rate $\dot{\gamma}$ between $\mathrm{De}=5 \times 10^{-5}$ and $\mathrm{De}=1 \times 10^{-2}$. It has been shown that the bubble model 170 represents accurately the Herschel-Bulkley rheology: $\tau=\tau_{y}+A \times \dot{\gamma}^{1 / 2}$ over this range (Langlois et al., 2008). If we keep the length and stiffness of the fibre fixed, respectively $N=50$ and $S=1$, we observe the variation shown in figure 6. As theoretically predicted by Jeffery (1922) for rigid ellipsoids and numerically observed by Yamamoto and Matsuoka (1993) for rigid particulate 175 rods in a Newtonian shear flow, we observe that the tumbling time decreases when $\dot{\gamma}$ or De increase. However, these studies predict that the tumbling time scales linearly with the inverse strain rate $\dot{\gamma}^{-1}$. In the case of a foam flow, the 
best fit to our data to a power law results in

$$
\frac{T_{t}}{\tau_{b}}=60.4 \times \mathrm{De}^{-0.85 \pm 0.03}
$$

which departs only slightly from the prediction (exponent -1) for slender objects.

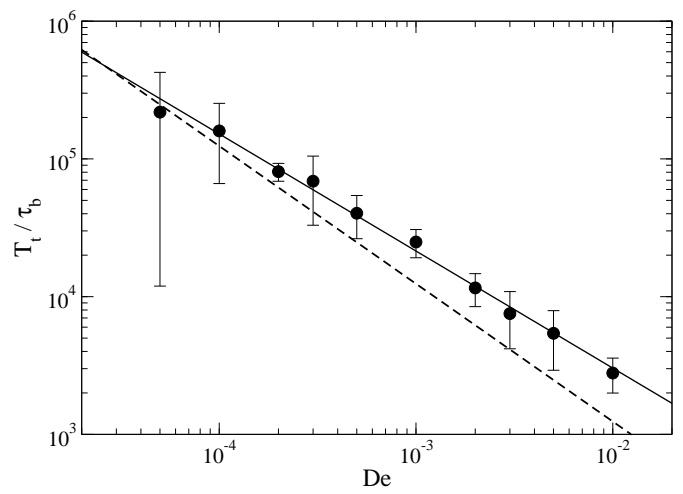

Figure 6: Tumbling time as a function of the dimensionless strain rate De, for a fibre of length $N=50$ and stiffness $S=1$. The plain line represents the best fit by a power law (see equation 9). The dashed line represents the best fit by an inverse function: $T_{t} / \tau_{b}=$ $12.4 \times \mathrm{De}^{-1}$.

180

\subsection{Influence of fibre length}

Since the scaling of the tumbling time with the strain rate is close to an inverse relationship, we now define the dimensionless tumbling time as $\tau_{t}=$ $\dot{\gamma} \times T_{t}$. In figure 7 , we plot this rescaled tumbling time as a function of the fibre length, all other parameters being kept constant. As can be observed, the longer the fibre, the faster it tumbles, which differs from results obtained by Yamamoto and Matsuoka (1993) for rigid fibers in the viscous shear flow of a Newtonian fluid (in accordance with predictions by Jeffery (1922)) and by Słowicka et al. (2012) for a single (but generally shorter) fibre in a Newtonian Poiseuille flow.

190 However, let us first note that the tumbling time quickly reaches a steady value $\tau_{0} \simeq 20$ when the fibre length exceeds 50 particles. Furthermore, very short fibres tend to exhibit chaotic dynamics with scarce random flips, which results in a very large dispersion in measured tumbling times (see error bars in figure 7). Let us insist on the fact that even if the tumbling time becomes independent of the fibre length, the detailed dynamics of the fibre can still differ. This can be evidenced by plotting the fraction of time $\xi$ that the fibre spends in a roughly straight geometry, defined as $\alpha<0.2$ (i.e., a configuration is considered as 


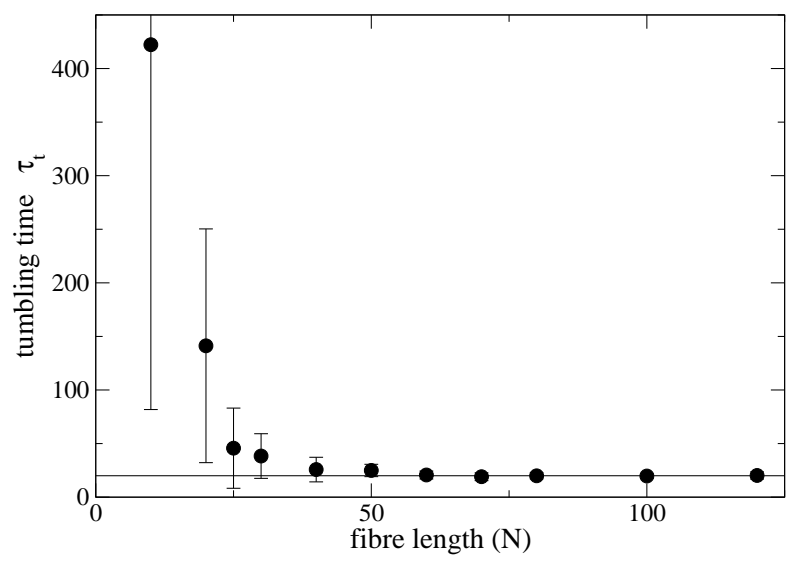

Figure 7: Dimensionless tumbling time $\tau_{t}=\dot{\gamma} T_{t}$ as a function of the length $N$ of the fibre, for De $=1.0 \times 10^{-3}$ and stiffness $S=1.0$. Vertical bars represent standard deviation of successive tumbling times. The straight line is the period $\tau_{0}=20$.

straight if $\left.L / L_{0}>0.8\right)$. As illustrated in figure $8, \xi$ is equal to 1 for very short fibres (which behave like rigid elongated particles) and continuously decreases with increasing fibre length. If, for short fibres, the flipping transition is almost instantaneous compared to the tumbling time, these two times become of the same order as the fibre gets longer, until the latter spends most of the time in a relatively coiled geometry.

In figure 9, we plot the orbits described by the two ends of the fibre in time, normalized by their distance at equilibrium. The orbit is close to a circle for the shortest fibre, which behaves like a rigid object. It is then quickly flattened in the transverse direction when the fibre gets longer. Let us remark, however, that in this case the streamwise distance between the two ends of the fibre does not represent the maximum streamwise extension of the fibre.

\subsection{Influence of fibre stiffness}

In figure 10 we plot the variation of tumbling time as a function of the dimensionless stiffness of a fibre made of $N=50$ particles, for $S=0.02$ to $S=35$. Considering the dispersion in observed tumbling times, we can conclude that in the range that we consider here, the stiffness of the fibre does not affect the tumbling time. This is partially consistent with the results of Yamamoto and Matsuoka (1993) which show that the tumbling time in a Newtonian fluid becomes constant if the fibre is rigid enough. As in the case of the influence of the fibre length, it is striking that the tumbling time remains almost invariant although the detailed dynamics of the flipping motion is different, as illustrated 


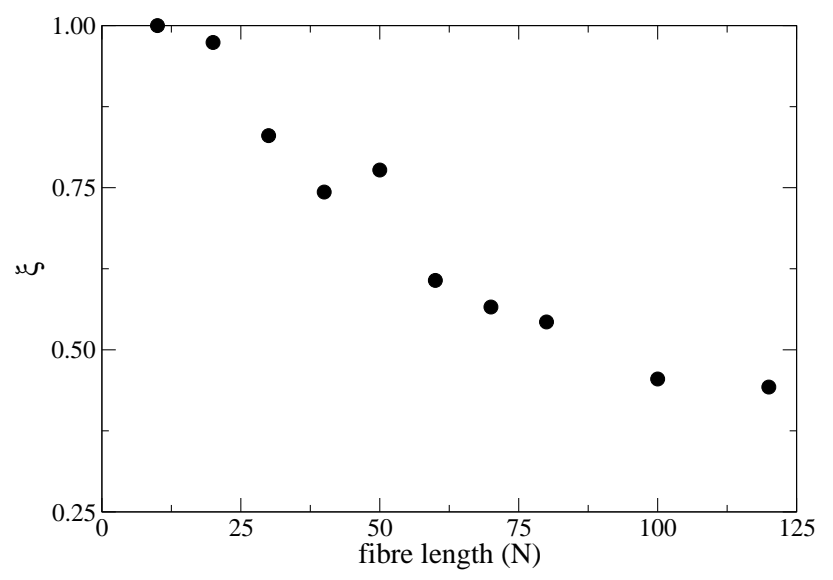

Figure 8: Fraction of the time, $\xi$, that the fibre spends in a relatively straight configuration $(\alpha<0.2)$, as a function of its length $N\left(S=1.0\right.$ and $\mathrm{De}=1.0 \times 10^{-3}$ as in figure 7$)$. Longer fibres tend to spend more time in a coiled configuration while flipping.

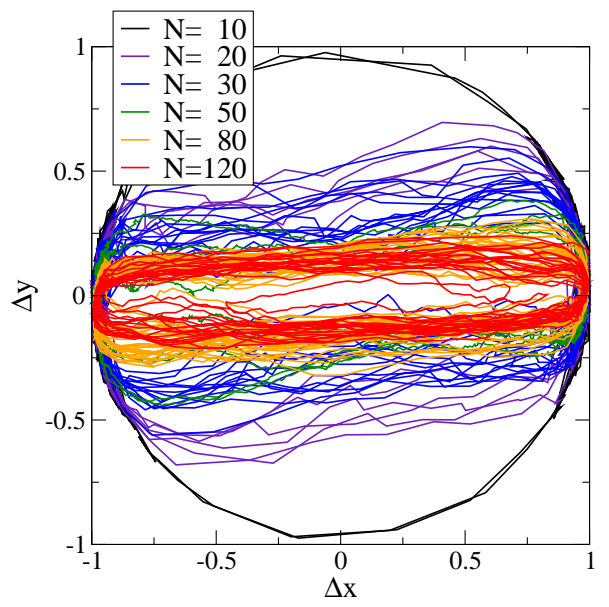

Figure 9: Transverse component versus streamwise component of the fibre end-to-end vector for different lengths of the fibre, each component being normalized by the fibre length. The longer the fibre, the flatter is the orbit. 


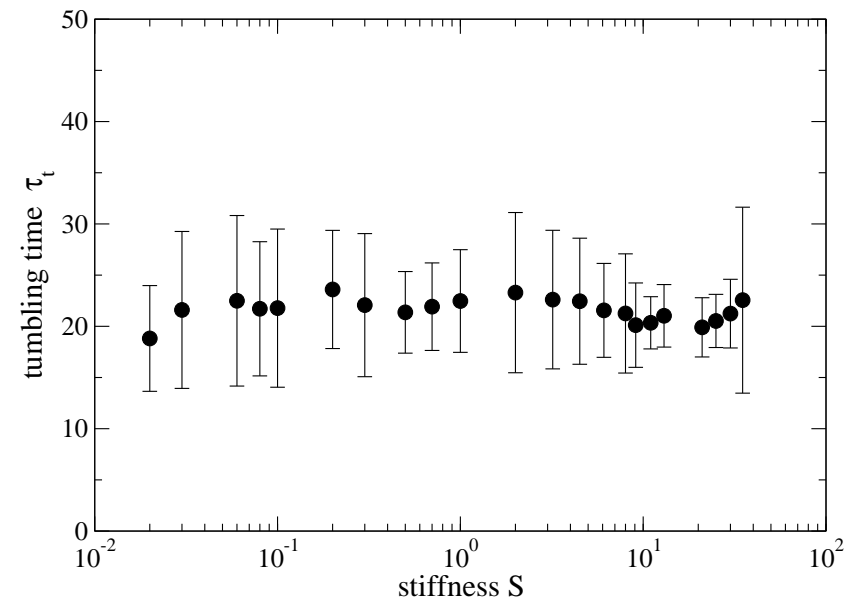

Figure 10: Tumbling time as a function of the dimensionless stiffness of the fibre, for a length $N=50$ and Deborah number $\mathrm{De}=10^{-3}$. Within the considered range, the tumbling time can be considered as independent of the fibre stiffness.

220 by figure 11 where we plot the standard deviation of the normalized transverse span of the fibre $\Delta y / L_{0}$. As can be seen, the average transverse extension of the fibre during the tumbling motion increases when its stiffness: flexible fibres are able to flip by taking sinuous shapes (which was called 'snake turn' by Forgacs and Mason (1959)), while stiffer fibres can only flip like rigid rods. In all cases, natural transverse velocity fluctuations of the bubbles constantly disturb the fibre from its straightened position aligned with the flow, which results in some parts of the fibre being accelerated. In the flexible case, as shown in figure 11, the typical transverse span is of the order of $\Delta y \sim L_{0} / 10$. This implies that the relative velocity of both ends of the fibre is of the order of $\Delta v \sim \dot{\gamma} \times L_{0} / 10$.

230 In order to flip, the fastest end of the fibre needs to be displaced by a distance $2 L_{0}$, which leads to a normalized tumbling time

$$
\tau_{t}=\dot{\gamma} T_{t}=\dot{\gamma} \times \frac{2 L_{0}}{\Delta v} \sim 20
$$

which is consistent with the observations (figures 6 and 10). In the rigid limit, the ends of the fibre undergo roughly circular orbits. In a Newtonian fluid, the tumbling time should be equal to half the period predicted by Jeffery (1922) for 235 rigid ellipsoids of aspect ratio $r$ : $\tau_{J}=\pi \times(r+1 / r)$. Extrapolating the effective aspect ratio $r^{*}$ of a cylindrical rods that behaves like an ellipsoidal rod of aspect ratio $r$ (Bretherton, 1962; Yamamoto and Matsuoka, 1993), we find $r^{*}=35$ for a fibre of length $N=50$, which corresponds to $\tau_{J}=110 \gg \tau_{0}$. Hence the actual tumbling time that we observe in our simulations is much shorter than predicted in a Newtonian fluid by Jeffery. 


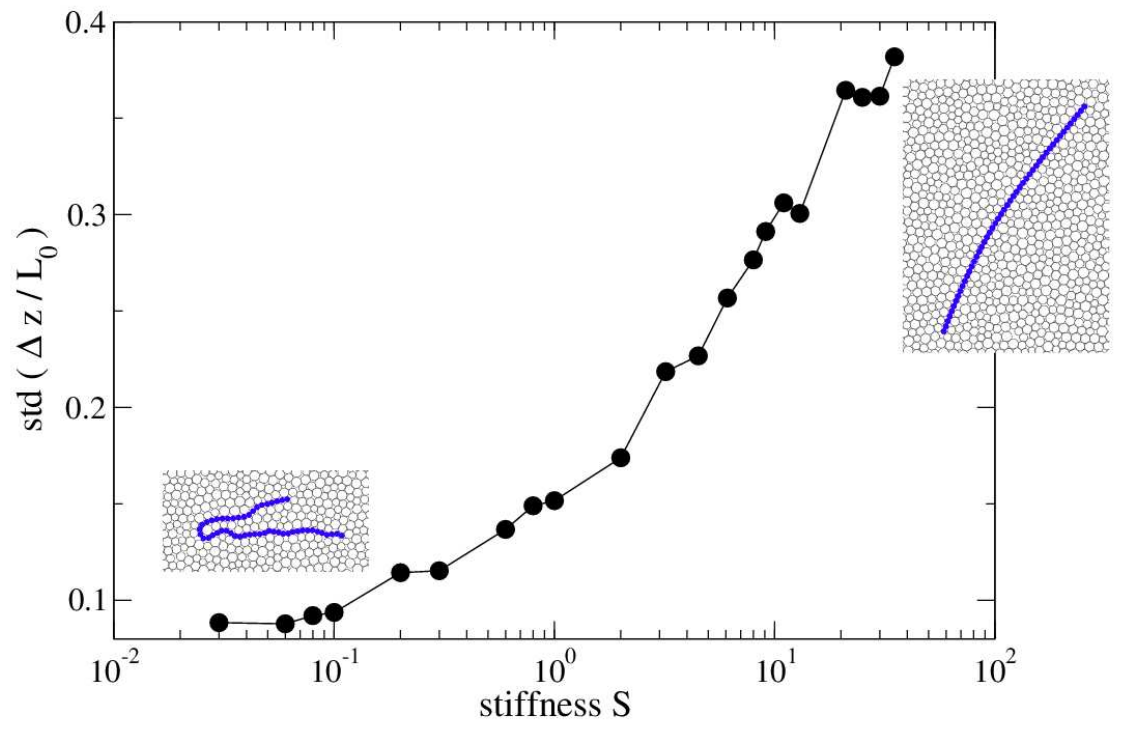

Figure 11: Standard deviation of the transverse span $\Delta y(t)$ of the fibre, normalized by the equilibrium length $L_{0}$, as a function of the stiffness $S$. Snapshots show the typical shape of the fibre during a flip in the limits $S \sim 0.1$ and $S \sim 20$. 


\subsection{Influence of gap width}

Finally, we varied the width of the gap between the two side-walls, and plotted the average tumbling time in figure 12. Let us note that the very large dispersion for the widest gap is in fact due to a small number of abnormally long times without any flip. However, considering the dispersion, it is difficult to assess that the width of the gap has any major influence on the tumbling time. Interestingly, it is also observed that the tumbling motion still occurs even when the width of the gap is smaller than the length of the fibre (if the latter is sufficiently flexible).

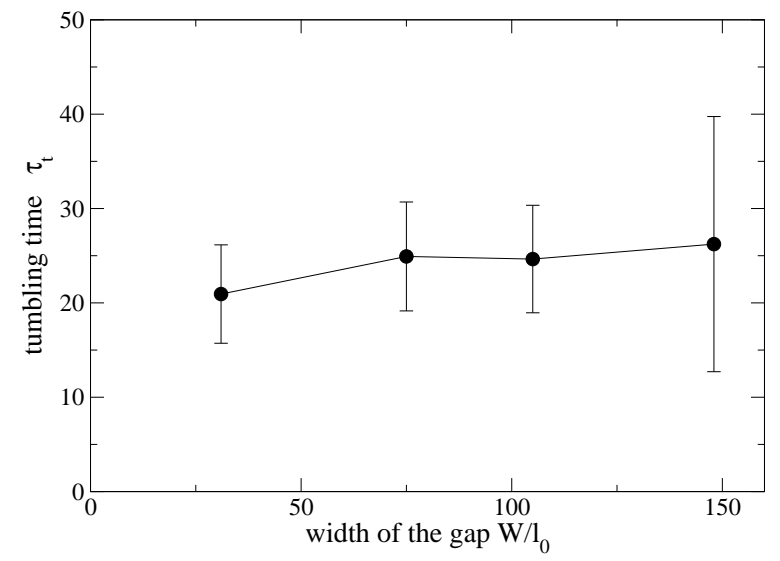

Figure 12: Dimensionless tumbling time $\tau_{t}=\dot{\gamma} T_{t}$ as a function of the width of the gap, normalized by the equilibrium length of the fibre $l_{0}$. Vertical bars represent standard deviation of successive tumbling times. We cannot conclude any particular influence of the gap width on the tumbling time.

\section{Conclusion}

In this article we have presented the first model of a fibre-laden foam. By combining the bubble model used in foam physics with a particulate model of a fibre classically used in simulations of fibre suspensions, we have investigated the dynamics of an individual flexible fibre within the shear flow of a 2D foam. Our simulations show that the tumbling time decreases as a power law of the strain rate, close to the inverse relationship already observed for fibres in a Newtonian shear flow. We also observe that the tumbling motion gets faster when the fibre gets longer, but becomes constant when the fibre length exceeds 50 bubble diameters. Interestingly, the tumbling time is also observed to be constant (with $\dot{\gamma} T_{t} \simeq 20$ ) when fibre stiffness is increased over 3 decades, though the detailed dynamics of the flipping motion is qualitatively different: short and rigid fibres describe roughly circular orbits and remain straightened, while long and flexible 
fibres spend most of the time in a coiled geometry and become sinuous when flipping. These results imply that using foam as a carrier fluid is not enough to keep fibres aligned in the direction of the flow. With this same issue in mind, we shall address the dynamics and interactions of multiple fibres in suspension within a foam, and the rheology of such a three-phase fluid, in a further article.

Recent experimental studies (Al-Qararah et al., 2015b) suggest that the rheology of a fibre-laden foam can be affected by the surface properties of the 270 fibres (which can be either smooth or rough). This effect could readily be incorporated in our model by adding a tunable attractive force between the fibre particles and adjacent bubbles, and between different fibres. Performing $2 \mathrm{D}$ experiments with model fibres in well-controlled flows would be useful to help tuning these numerical ingredients. Finally, let us note that the model can 275 also conveniently be extended to non-homogeneous strain rates (e.g. for a pipe flow) and, by adding twisting forces, to a suspension of fibres in a $3 \mathrm{D}$ foam.

Acknowledgments. This work has emanated from research supported in part by a research grant from Science Foundation Ireland (SFI) under grant number 13/IA/1926. This article is based upon work from COST Actions MP1106 'Smart and green interfaces' and MP1305 'Flowing matter', supported by COST (European Cooperation in Science and Technology). We also acknowledge the support of the European Space Agency ESA, Project microG-Foam, AO-99-075 and contract 4000115113, 'Soft Matter Dynamics'. We would like to thank F. Dunne for stimulating discussions.

\section{References}

\section{References}

Al-Qararah, A.M., Ekman, A., Hjelt, T., Ketoja, J.A., Kiiskinen, H., Koponen, A., Timonen, J., 2015a. A unique microstructure of the fiber networks deposited from foam-fiber suspensions. Colloids and Surfaces A: Physicochemical and Engineering Aspects 482, 544-553. doi:10.1016/j.colsurfa.2015.07.010.

Al-Qararah, A.M., Hjelt, T., Koponen, A., Harlin, A., Ketoja, J.A., 2013. Bubble size and air content of wet fibre foams in axial mixing with macroinstabilities. Colloids and Surfaces A: Physicochemical and Engineering Aspects 436, 1130-1139. doi:10.1016/j.colsurfa.2013.08.051.

295 Al-Qararah, A.M., Hjelt, T., Koponen, A., Harlin, A., Ketoja, J.A., 2015b. Response of wet foam to fibre mixing. Colloids and Surfaces A: Physicochemical and Engineering Aspects 467, 97-106. doi:10.1016/j.colsurfa.2014.11.034.

Bretherton, F.P., 1962. The motion of rigid particles in a shear flow at low Reynolds number. Journal of Fluid Mechanics 14, 284-304.

300 Cantat, I., Cohen-Addad, S., Elias, F., Graner, F., Höhler, R., Pitois, O., Rouyer, F., Saint-Jalmes, A., 2013. Foams: structure and dynamics. OUP Oxford. 
Durian, D., 1995. Foam mechanics at the bubble scale. Phys. Rev. Lett. 75, 4780-4783. doi:10.1103/PhysRevLett.75.4780.

A., Piasecki, T., Słowicka, A.M., Misbah, C., Wajnryb, E., Ekiel-Jezewska, M.L., 2016. Dynamics of flexible fibers and vesicles in Poiseuille flow at low Reynolds number. Soft Matter 42, 7307-7323. doi:10.1039/C6SM00819D.

Forgacs, O., Mason, S., 1959. Particle motions in sheared suspensions: IX. spin and deformation of threadlike particles. Journal of Colloid Science 14, $457-472$.

Gauger, E., Stark, H., 2006. Numerical study of a microscopic artificial swimmer. Physical Review E 74, 021907. doi:10.1103/PhysRevE.74.021907.

Haffner, B., Dunne, F.F., Burke, S.R., Hutzler, S., 2017. Ageing of fibre-laden aqueous foams. Cellulose 24, 231-239. doi:10.1007/s10570-016-1100-1.

Jäsberg, A., Selenius, P., Koponen, A., 2015. Experimental results on the flow rheology of fiber-laden aqueous foams. Colloids and Surfaces A: Physicochemical and Engineering Aspects 473, 147-155. doi:10.1016/j.colsurfa.2014.11.041.

Jeffery, G.B., 1922. The motion of ellipsoidal particles immersed in a viscous fluid. Proceedings of the Royal Society of London A: Mathematical, Physical and Engineering Sciences 102, 161-179.

Langlois, V.J., 2014. The two-dimensional flow of a foam through a constriction: Insights from the bubble model. Journal of Rheology 58, 799-818. doi: $10.1122 / 1.4872058$.

325 Langlois, V.J., Hutzler, S., Weaire, D., 2008. Rheological properties of the soft-disk model of two-dimensional foams. Phys. Rev. E 78, 021401. doi:10.1103/PhysRevE.78.021401.

Lindner, A., Shelley, M., 2015. Elastic fibers in flows. Royal Society of Chemistry, Cambridge, U.-K. p. 168.

330 Lindström, S.B., Uesaka, T., 2007. Simulation of the motion of flexible fibers in viscous fluid flow. Physics of Fluids 19, 0-16. doi:10.1063/1.2778937.

Pöschel, T., Schwager, T., 2005. Computational granular dynamics: models and algorithms. Springer-Verlag, Berlin.

Ross, R.F., Klingenberg, D.J., 1997. Dynamic simulation of flexible fibers com335 posed of linked rigid bodies. The Journal of Chemical Physics 106, 2949-2960. doi:10.1063/1.473067.

Sexton, M.B., Möbius, M.E., Hutzler, S., 2011. Bubble dynamics and rheology in sheared two-dimensional foams. Soft Matter 7, 11252-11258. doi:10.1039/C1SM06445B. 
Słowicka, A.M., Ekiel-Jezewska, M.L., Sadlej, K., Wajnryb, E., 2012. Dynamics of fibers in a wide microchannel. Journal of Chemical Physics 136, 0-8. doi:10.1063/1.3678852.

Słowicka, A.M., Wajnryb, E., Ekiel-Jezewska, M.L., 2015. Dynamics of flexible fibers in shear flow. The Journal of Chemical Physics 143, 124904. doi:10.1063/1.4931598.

Smith, M., Punton, V., Rixson, A., 1974. Structure and properties of paper formed by a foaming process. Tappi $57,107-111$.

Subramanian, G., Koch, D.L., 2005. Inertial effects on fibre motion in simple shear flow. Journal of Fluid Mechanics 535, 383-414. doi:10.1017/S0022112005004829.

Switzer, L.H., Klingenberg, D.J., 2003. Rheology of sheared flexible fiber suspensions via fiber-level simulations. Journal of Rheology 47, 759. doi:10.1122/1.1566034.

Wandersman, E., Quennouz, N., Fermigier, M., Lindner, A., Du Roure, 355 O., 2010. Buckled in translation. Soft matter 6, 5715-5719. doi:10.1039/C0SM00132E.

Weaire, D., Hutzler, S., 1999. The Physics of Foams. Oxford University Press, Oxford.

Whyte, D., Haffner, B., Hjelt, A., Hutzler, S., 2017. The interactions of fibres with soap films. to appear in Coll. Surf. A .

Yamamoto, S., Matsuoka, T., 1993. A method for dynamic simulation of rigid and flexible fibers in a flow field. The Journal of Chemical Physics 98, 644650. doi:10.1063/1.464607.

Yamamoto, S., Matsuoka, T., 1996. Dynamic simulation of microstructure and rheology of fiber suspensions. Polymer Engineering \& Science 36, 2396-2403. doi:10.1002/pen.10638. 
Recently there has been a renewed interest in using foamy suspensions of wood fibres as

a carrier fluid in papermaking but there is a lack of fundamental

understanding of the dynamics of such a three-phase system.

In this article we propose a numerical model for the dynamics of an individual flexible fibre within a flowing foam, based

on discrete-element methods. As is observed in a Newtonian shear flow, we observe that the fibre systematically experiences a tumbling instability: the disordered motion of bubbles

cannot prevent the pseudo-periodical flip of the fibre. Our simulations show that the tumbling time decreases almost as the inverse of the strain rate. It also decays when the fibre

length is increased, though for long enough fibres it reaches a constant value. Similarly the tumbling time is also surprisingly independent of the stiffness of the fibre.

Because of their tumbling motion, long and flexible fibres spend most of the time in a coiled geometry. This would imply that using foam as a carrier fluid is not enough to keep fibres aligned with the flow. However, further refinements of the model will need to be considered to arrive at firm conclusions regarding alignment. 